\title{
A Retrospective Match Controlled Study of Supersaturated Calcium Phosphate Oral Rinse vs. Supportive Care for Radiation Induced Oral Mucositis*
}

\author{
Curtis T. Miyamoto, Jessica Wobb, Bizhan Micaily, Shidong Li, Mohan P. Achary \\ Department of Radiation Oncology, Temple University Hospital and School of Medicine, Philadelphia, USA. \\ Email: miyamoc@tuhs.temple.edu, ${ }^{*}$ achary@temple.edu
}

Received August $9^{\text {th }}, 2012$; revised September 11 ${ }^{\text {th }}, 2012$; accepted September $22^{\text {nd }}, 2012$

\begin{abstract}
Oral mucositis is a common morbidity induced by radiation therapy and chemo-radiotherapy for head and neck malignancies. This often results in treatment delays, premature treatment cessation and increased cost. New treatments of oral mucositis are emerging but effective remedies remain limited. Between February 2007 and May 2008, 21 patients with head and neck malignancies were treated with a supersaturated calcium phosphate oral rinse (Caphosol) that was performed for 4 - 10 times daily, each consisting of two one-minute rinses. There were 21 matched patients who received supportive care without the oral rinse. All patients in the two groups were treated with intensity modulated radiation therapy (IMRT) for average prescription doses of $66 \mathrm{~Gy}$ in 33 fractions. The effects of this rinse vs. supportive care on mucositis, PEG tube requirements, hospitalization, Xerostomia, analgesic requirements and weight was respectively evaluated. We have observed statistically significant decreases in the incidence of radiation induced oral mucositis $(\mathrm{p}=$ $0.0002)$ with WHO grade $3(38 \%$ vs. $52 \%$ ) and 4 mucositis ( $0 \%$ vs. $19 \%)$, need for PEG tube placement ( $33 \%$ vs. $57 \%$ ) and hospitalization $(0 \%$ vs $19 \%)$. There was also a favorable impact on the incidence of grade $3(29 \%$ vs. $43 \%)$ Xerostomia in the treated patients but it was statistically insignificant $(\mathrm{p}=0.58)$. Incremental costs related to treatment of oral mucositis were reduced considerably. Our data suggest that use of a supersaturated calcium phosphate oral rinse is an effective treatment for mucositis in patients undergoing IMRT. It may also be helpful in the reduction of hospitalization and PEG tube requirements.
\end{abstract}

Keywords: Oral Rinse; Mucositis; Radiation; Chemotherapy; Head and Neck

\section{Introduction}

Oral mucositis (OM) can affect up to $100 \%$ of patients with malignancies of the head and neck receiving radiation treatment and up to $80 \%$ receiving chemotherapy and hematopoietic stem cell transplantation (HSCT) [1]. It is a leading cause of treatment-limiting toxicity [2]. Mucositis continues to be a major challenge for healthcare providers with severe cases adding significantly to health care costs $[3,4]$.

Mucositis is defined as painful inflammation and ulceration of the mucous membranes of the oral and gastrointestinal tract $[5,6]$. As a result of cell death in reaction to chemo- or radiotherapy, the mucosal lining of the mouth becomes thin, may slough off and then become red, inflamed and ulcerated. Oral mucositis can be severely painful and have a significant impact on a patient's quality of life. The degree of pain is usually re-

\footnotetext{
*Conflict of interest: Speakers Bureau for EUSA.

\#Corresponding author.
}

lated to the extent of the tissue damage [7]. Pain is often described as a burning sensation accompanied by reddening. Due to pain, the patient may experience trouble speaking, eating, or even opening the mouth [8].

Pain and loss of taste perception makes it more difficult to eat, which leads to weight loss. Ulcers may act as a site for local infection and a portal of entry for oral flora that, in some instances, may cause septice- mia [5]. For patients receiving standard dose chemother- apy the incidence and severity of OM varies considerably as a function of the specific agent and schedule administered. Among chemotherapy patients that experience Grade 3 - 4 OM, more than $62 \%$ require hospital admission or extended hospitalization for total parenteral nutrition, intravenous analgesia, and intravenous antibiotics and $70 \%$ require feeding tubes [7]. Infectious episodes are twice as common during cycles of chemotherapy with oral and/or GI mucositis than those without (73\% vs. $36 \% ; \mathrm{p}<0.001)$. Similarly, dose reductions in the following cycle of chemotherapy are twice as likely after 
cycles with oral and/or GI mucositis than after cycles without mucositis ( $23 \%$ vs. $11 \%$; $<<0.0001)$ [2,9].

Diagnosis is based largely on the symptoms the patient is experiencing and the appearance of the tissues of the mouth. Red burn-like sores or ulcers throughout the mouth is enough to diagnose mucositis. It is graded on a scale of 0 to 4 according to the World Health Organization (WHO) grading scale. In grade $3 \mathrm{OM}$, the patient is unable to eat solid food, and in grade 4, the patient is unable to consume liquids as well [10].

Among patients undergoing head and neck radiotherapy, pain and decreased oral function may persist long after the conclusion of therapy. Fractionated radiation dosage increases the risk of severe mucositis to $>70 \%$ of patients in most trials. Mucositis associated with radiotherapy usually appears at the end of the second week of treatment and may last for six to eight weeks [11].

Two recent studies $[3,4]$ have examined the costs of supportive care associated with the development of OM in patients with head and neck cancers treated with radiation and chemotherapy. Elting [3] reported the per patient incremental cost of Grade $1-2 \mathrm{OM}$ to be approximately $\$ 1700$ and that of Grade $3-4 \mathrm{OM}$ to be approximately $\$ 6000$. Nonzee's [4] analysis which was limited to severe OM (Grade 3 - 4) found the incremental cost per patient to be approximately $\$ 17,000$. The differences in costs between these analyses are related primarily to a shorter duration of hospitalization for the patients in the Elting study, potential explanations for which are discussed in each of the publications $[3,4]$.

Current clinical management of mucositis is mainly supportive, with a concentration on reducing risk factors and symptomatic relief. The European Society of Medical Oncology (ESOM) and Multinational Association of Supportive Care in Cancer and International Society of Oral Oncology (MASCC/ISOO) have recently published guidelines for treating mucositis [12]. Recommendations focus mainly on preventative measures, including highquality clinical practice, good oral hygiene, nutritional support, and adequate pain management. These include using bland oral rinses, topical anesthetics, mucosal coating agents, and analgesic rinses.

Several promising therapeutic agents are currently under review and being assessed for evidence-based evaluation. Experimental therapies, including the use of various cytokines and growth factors, vitamin and mineral supplementation, and cryotherapy, have been reported, although there remains to be no definitive treatment regimen [13-20]. A Cochrane Database systemic review addressing this issue identified several intervenetions had some benefit at preventing or reducing the severity of mucositis associated with cancer treatment, although they call for more trials with sufficient numbers of participants to perform subgroup analyses [21].

Caphosol, a supersaturated calcium phosphate rinse (SCPR) (EUSA Pharma (USA), Inc., Langhorne, PA), has recently been identified as a possible preventative measure against $\mathrm{OM}$ for patients receiving chemo- and radiation therapy [22-29]. High concentrations of $\mathrm{Ca}^{2+}$ and PO4 in caphosol are known to be valuable and beneficial supplemental source for damaged oral mucosal surfaces. However, the molecular mode of action of the repair by caphosol is not clear. Papas, et al. [27] hypothesized that high concentration of $\mathrm{Ca}^{2+}$ ions, diffuse into intercellular spaces of the epithelium and permeate the mucosal lesions in mucositis. Thus these $\mathrm{Ca}^{2+}$ ions may play an important role in the inflammatory process, the blood clotting cascade and tissue repair. It is also suggested that inorganic phosphate is essential for the biochemical processes involved in mucositis. There have been several studies evaluating the use of SCPR to reduce the incidence, severity and duration of mucositis in cancer patients undergoing chemo- and/or radiotherapy with and without haematopoietic stem cell transplantation (HSCT) treatments [22-29].

The purpose of this study is to look at outcomes of patients diagnosed with head and neck malignancies, subsequently treated with radiation therapy, and the impact of SCPR on symptom-related outcomes, including oral mucositis and other associated comorbidities.

\section{Materials and Methods}

From February 2007 to May 2008, twenty-one patients were subjected to a SCPR treatment regimen for the prevention and management of radiation induced mucositis. Consistent with the approved labeling, patients were instructed to rinse their mouths for one minute two times with SCPR 4 - 10 times daily. The patients began use of the SCPR the first day of cancer treatment and continued until the treatment was completed. All patients were treated with intensity-modulated radiation therapy (IMRT). The details of the inclusion and exclusion criteria, types of cancers included as well as radiation treatment regimen used in this study are listed below:

The inclusion criteria:

1) Patients had to have IMRT fields that included evaluable oral mucosa.

2) Patients had a minimum of $4500 \mathrm{cGy}$ to the oral mu- cosa.

3) Patients used Caphosol at least daily.

4) Patients had their mucous membranes evaluated at least once a week.

5) Patients had to have a corresponding case matched patient.

The exclusion criteria:

1) Patients who did not have a corresponding case 
match patient.

2) Patients who did not have radiation therapy including the oral mucosa or achieve a minimum total dose of $4500 \mathrm{cGy}$.

3) Did not use Caphosol at least daily.

4) Did not have weekly evaluation of the oral mucosa.

The control group consisted of case matched patients who did not use Caphosol but had to have the following criteria:

1) Patients had to have IMRT fields that included evaluable oral mucosa.

2) Patients had a minimum of $4500 \mathrm{cGy}$ to the oral mu- cosa.

3) Patients had their mucous membranes evaluated at least once a week.

4) Patients had to have a corresponding case matched patient who used Caphosol.

5) When indicated, patients also had chemotherapy.

Types of Cancer included in this study:

Control group: Sites: glottis 6 patients, parotid 2 patients, oropharynx 8 patients, maxillary sinus 1 patient, supraglottic larynx 4 patients.

Treatment group: glottis 2 patients, parotid 1 patient, oropharynx 5 patients, floor of mouth 4 patients, pyriform sinus 3 patients, nasopharynx 2 patients, unknown primary 1 patient, metastatic disease to the parotid 1 patient, hypopharynx 1 patient and supraglottic larynx 1 patient.

Radiation treatment regimen:

Control group: 180 - $225 \mathrm{cGy} /$ fraction for 20 patients and $285 \mathrm{cGy} /$ fraction for 1 patient. Total doses: $7 \mathrm{pa}-$ tients $>7000 \mathrm{cGy}, 11$ patients $>6000 \mathrm{cGy}-7000 \mathrm{cGy}$ and 3 patients $<6000 \mathrm{cGy}$.

Caphosol group: $180-225 \mathrm{cGy} /$ fraction for all patients. Total doses: 10 patients $>7000 \mathrm{cGy}, 8$ patients $>6000$ cGy - 7000 cGy and 3 patients $<6000$ cGy.

The average prescription doses for both groups are 200 cGy per fraction for total 33 fractions. Treatment planning was carried out using the Pinnacle ${ }^{3 \mathrm{TM}}$ treatment planning system by ADAC and dose to regions of interest were quantified.

To assess the effectiveness of this new treatment regimen a retrospective chart review was carried out including all patients using SCPR during radiation therapy. SCPR treated patients were match-controlled with patients receiving standard supportive care. All patients were treated at Temple University Hospital, Department of Radiation Oncology. Match control patients were treated during the same time period. They were matched by age, radiation technique, fractionation, total dose and evaluable mucosa in the radiation fields. Control patients received other supportive regimens including analgesics, "magic mouthwash" (diphenhydramine, bismuth sub- salicylate, and viscous lidocaine), salt and soda rinses, percutaneous endoscopic gastrostomy (PEG) tubes, etc.

Data collected included patient demographics and clinical characteristics (age, gender, treatment dates, cancer diagnosis), daily fraction size, total dose, amount of radiation to each parotid gland, maximum grade of mucositis and Xerostomia acquired during treatment, weight loss, whether analgesics were prescribed during radiation therapy, need for PEG tube, and incidence and duration of mucositis related hospitalization during treatment. Mucositis and Xerostomia were graded according to physician's physical exam and clinical assessment based on the WHO grading system [10].

While comparing the two data sets of the patient groups, the probability (p-value) of Student's t-Test with paired and two tails was calculated for determination of the significant differences for the evaluated effects on $\mathrm{OM}$ and Xerostomia. If the $\mathrm{p}$-value is $<0.05$, the effect of Caphosol is considered to be significant for a given group of patients.

The cost of OM was estimated using two independent previously reported cost models $[3,4]$ each of which reported incremental costs related to mucositis based on severity (Grade 1 - 2 and Grade 3 - 4 for the Elting model and Grade 3 - 4 for the Nonzee model). Costs for each model were converted to 2009 dollars assuming a $5 \%$ annual rate of inflation. The impact of treatment with SCPR on costs related to OM was evaluated by comparison of the magnitude of the incremental cost per patient for the treated and control groups based on each of the models.

\section{Results}

\subsection{Demographics}

Demographic and clinical characteristics are presented in Table 1. SCPR treated and control patients were very similar with regard to age, sex and cancer diagnosis. Both groups received similar amounts of radiation with regard to both total dose and daily fractions. Concomitant chemotherapy was received by nine patients in the SCPR treated group ( 2 patients with cetuximab, 2 patients cisplatin, 1 patient 5-fluorouracil and cisplatin, 2 patients carboplatin and paclitaxol, 1 patient paclitaxol and 1 patient carboplatin and paclitaxol) and 13 patients in the matched control group (6 patients cisplatin, 4 patients carboplatin and paclitaxol, 2 patients with cetuximab and 1 patient with gemcitabine, cetuximab, carboplatin and paclitaxel). Two patients in the matched control group received only one cycle of chemotherapy.

\subsection{Oral Mucositis and Xerostomia}

As compared to the control patients, a greater proportion 
Table 1. Demographics and treatment characteristics in SCPR and control groups.

\begin{tabular}{lcc}
\hline Characteristics & $\begin{array}{c}\text { SCPR group } \\
\text { No. (\%) }\end{array}$ & $\begin{array}{c}\text { Control group } \\
\text { No. (\%) }\end{array}$ \\
\hline Age (median, range) & 63 & 61 \\
Sex & $36-95$ & $46-74$ \\
Male & $14(67 \%)$ & $15(61 \%)$ \\
Female & $7(33 \%)$ & $6(29 \%)$ \\
Cancer Dx & 4 & 0 \\
Oral & 2 & 1 \\
Nasopharynx & 7 & 9 \\
Oropharynx & 4 & 1 \\
Hypopharynx & 4 & 10 \\
Larynx & 204 & 207 \\
Dose fraction, cGy per day & $6770 \pm 750$ & $6780 \pm 600$ \\
Total dose, cGy & $9(43 \%)$ & $13(62 \%)^{*}$ \\
Adjuvant chemotherapy &
\end{tabular}

${ }^{*}$ Two patients received one cycle of chemotherapy.

of the SCPR treated patients, experienced mild (Grade 0 or 1) OM ( $48 \%$ vs. $29 \%)$ and a smaller proportion experienced severe (Grade 3 or 4$)$ OM (38\% vs. $71 \%$ ). This was a dose-dependent finding; all patients diagnosed with Grade 4 OM received at least 7400 cGy during radiation therapy.

There were similar incidences of Xerostomia between groups during therapy ( $62 \%$ of the treated patients had no more than Grade 1 Xerostomia and 57\% of control patients had similar results), although the treated group received on average $157 \%$ more radiation to their parotid glands than the control group (3090 cGy vs. 1950 cGy, respectively). All results are presented in Table 2.

OM data of the two groups of individual patients have the p-value of 0.0002 in the Student's t-Test which indicates the statistically significant improvement with the use of SCPR. On the other hand, Xerostomia data showed the p-value of 0.58 which indicates that the difference between the two groups is insignificant.

\subsection{Cancer Treatment Related Morbidities}

Only $33 \%$ of treated patients required PEG tubes for nutrition while $57 \%$ of control patients needed them. In addition, four of the control patients $(19 \%)$ were admitted for in-patient treatment during radiation therapy for failure to thrive; however, no patients receiving SCPR treatment required hospitalization. The hospitalized patients were admitted for 6, 9, 15 and 16 days. In all cases dysphagia was a significant component for admission.
Patients were admitted during treatment in three cases and immediately after treatment in one case. There were no differences between groups in regards to weight loss or incidence of prescribed analgesics during treatment (Table 3). No patients developed secondary effects from the oral rinse.

\subsection{Costs Related to Oral Mucositis}

Incremental per patient costs related to the treatment of oral mucositis were $\$ 5306$ - $\$ 14,882$ for control patients depending on the cost model employed (Table 4). For the SCPR treated patients the corresponding values were $\$ 3584$ - \$7965. This resulted in a savings of $\$ 1722$ $\$ 6917$ per patient for the treated patients.

\section{Discussion}

Radiation and chemotherapy induced mucositis continues

Table 2. Rates of oral mucositis and Xerostomia in SCPR and control groups.

\begin{tabular}{ccc}
\hline & $\begin{array}{c}\text { SCPR group } \\
\text { No. (\%) }\end{array}$ & $\begin{array}{c}\text { Control group } \\
\text { No. (\%) }\end{array}$ \\
\hline Oral mucositis & & \\
Grade 0 & $3(14 \%)$ & $2(10 \%)$ \\
Grade 1 & $7(33 \%)$ & $4(19 \%)$ \\
Grade 2 & $3(14 \%)$ & 0 \\
Grade 3 & $8(38 \%)$ & $11(52 \%)$ \\
Grade 4 & 0 & $4(19 \%)$ \\
Xerostomia & & $11(52 \%)$ \\
Grade 0 & $5(24 \%)$ & $1(5 \%)$ \\
Grade 1 & $8(38 \%)$ & 0 \\
Grade 2 & $2(10 \%)$ & $9(43 \%)$ \\
Grade 3 & $6(29 \%)$ & 0 \\
Grade 4 & 0 & \\
\hline
\end{tabular}

Table 3. Cancer treatment related morbidities in SCPR and control groups.

\begin{tabular}{lcc}
\hline Outcome & $\begin{array}{c}\text { SCPR group } \\
\text { No. (\%) }\end{array}$ & $\begin{array}{c}\text { Control group } \\
\text { No. }(\%)\end{array}$ \\
\hline Weight loss (lbs) & 9.6 & 8.4 \\
Need for analgesics & $10(48 \%)$ & $11(55 \%)$ \\
PEG requirement & $7(33 \%)$ & $12(57 \%)$ \\
OM related hospitalization & 0 & $4(19 \%)$ \\
\hline
\end{tabular}

Table 4. Estimated per patient incremental costs related to oral mucositis (\$).

\begin{tabular}{cccc}
\hline Cost model & Control patients & Treated patients & Cost difference \\
\hline Elting & 5306 & 3584 & 1722 \\
Nonzee & 14,882 & 7965 & 6917 \\
\hline
\end{tabular}


to be one of the most debilitating and dose limiting secondary effects of contemporary management of patients with head and neck malignancies. It can result in treatment delays, early stoppage of treatment, hospitalization, malnutrition, dehydration and death. There have been few studies demonstrating effective treatments. SCPR is an approved treatment for radiation and chemotherapy induced mucositis. Papas, et al., published two studies that demonstrated that SCPR treatment was well tolerated and reduced the incidence of oral mucositis in patients receiving hematopoietic stem-cell transplantation and head and neck radiotherapy $[25,26]$. A subsequent placebo controlled trial in transplant patients confirmed these positive results and showed that the severity of oral mucositis was significantly lower in the group treated with SCPR in comparison to the control group [27]. Similar studies by Wasko-Grabowska, et al. [28,29] reiterated that SCPR reduces in the incidence, severity and duration of mucositis as well as decrease in the days of taking pain killers among the patients treated with BEAM (BCNU [carmustine] + etoposide + ARA-C [cytarabine] + melphalan) but not in MEL 200 (melphalan) regimen. However, these studies were conducted in single centers and only in transplant patients, although the results are similar to the previous retrospective studies.

An open label multicenter observational registry was conducted that included patients with a variety of primary malignancies who received radiotherapy and/or chemotherapy with the risk of developing OM. Oral mucositis was assessed using the NCI common toxicity criteria. Consistent with our findings, among the 68 head and neck cancer patients reported in that study [23] no patient experienced Grade 4 functional oral mucositis and only a single patient experienced Grade 4 clinical oral mucositis.

In this study we retrospectively reviewed patients treated with IMRT for head and neck malignancies and compared them with match controlled patients receiving essentially the same regimen. We found clinically meaningful decreases in the incidence of radiation induced WHO grade 3 (38\% vs. $52 \%)$ and $4(0 \%$ vs. $19 \%)$ mucositis. These reductions in high grade mucositis were accompanied by similar reductions in the need for PEG tube placement $(33 \%$ vs. $57 \%)$ and hospitalization ( $0 \%$ vs. $19 \%)$. We also observed a reduction in the development of grade 3 Xerostomia ( $29 \%$ vs. $43 \%$ ) in Caphosol treatment group in spite of the fact that these patients received a $157 \%$ higher dose of radiation therapy to the parotid glands. It is not clear whether this difference was due simply to the wetting action associated with the oral rinse or whether use of the rinse may have an, as of yet unexplained, protective effect on the major/minor salivary glands. However, the observed effect was found to be statisticcally insignificant $(p=0.58)$ in our study. Probably a study with a larger cohort of patients may be required to validate this interesting observation. If this finding proves to be true, the reduction in Xerostomia will definitely provide an additional benefit to improve the patient's quality of life during treatment.

Our OM data compares favorably with the results of several recently presented studies. In a multinational, randomized, double-blind, placebo-controlled study by $\mathrm{Wu}$, et al. [30] compared the therapeutic effect of recombinant human epidermal growth factor (EGF) on mucositis in patients with head and neck cancer undergoing radiotherapy with or without chemotherapy. This study showed that the EGF $(50 \mu \mathrm{g} / \mathrm{mL})$ treated patients had a reduced incidence of severe OM (64\% response with EGF vs. 37\% with placebo) compared to controls.

Another multinational, randomized, double blind, placebo controlled, phase 3 trial by Le, et al. [31], palifer$\min (180 \mu \mathrm{g} / \mathrm{kg})$ was given intravenously 3 days before the start of chemo-radiotherapy (2 Gy/fraction to 7000 cGy with concurrent cisplatin at $100 \mathrm{mg} / \mathrm{m}^{2}$ ) followed once weekly until completion of treatment. This study showed that the incidence of severe mucositis was reduced from $69 \%$ for the placebo group to $54 \%$ for the palifermin group $(p=0.041)$. Although caution needs to be taken when comparing prospective randomized data with retrospective data, our findings provide a strong rationale for a prospective evaluation of the efficacy of this agent in the prevention and treatment of OM in patients receiving radiation and chemotherapy for malignancies of the head and neck.

The rates of both overall and severe OM observed for the control patients in the current study were very similar to those reported in the studies which provided the basis for estimating the impact on treatment costs. Elting [3] reported an overall rate of $\mathrm{OM}$ of $91 \%$ with a rate of severe (Grade 3 - 4) OM of $66 \%$ as compared to corresponding rates of $90 \%$ and $71 \%$, respectively for the control patients in the current study. Similarly, the rate of severe OM (the only measure reported) in the Nonzee study was $70 \%[4]$.

The reductions in the severity of OM for the SCPR treated patients resulted in an estimated cost savings of $\$ 1722$ - \$6917 per patient. The reductions in need for hospitalization and PEG tubes in the treated patients in the current study corroborated the cost saving estimates derived from the previously published cost models based on OM severity. The difference between models in costs associated with mucositis derives primarily from differences in the duration of hospitalization which was 15 days in the Nonzee study as compared to seven days in the Elting study. The duration of hospitalization in our study (12 days) was closer to that reported by Nonzee 
indicating that the per patient cost savings associated with SCPR treatment would be expected to be closer to the upper end of the range given above based on our findings.

In conclusion, even with the limited number of 42 patients in our match-controlled study, the reduction in the occurrence of $\mathrm{OM}$ has been proved to be statistically significant in Caphosol treated group of patients. Furthermore, the Caphosol oral rinse was well tolerated (no patients developed secondary effects) and patients were able to carry it with them in individually dosed containers allowing them to be compliant with the treatment.

\section{REFERENCES}

[1] E. B. Rubenstein, D. E. Peterson, M. Schubert, D. Keefe, D. McGuire, J. Epstein, L. S. Elting, P. C. Fox, C. Cooksley and S. T. Sonis, "Clinical Practice Guidelines for the Prevention and Treatment of Cancer Therapy-Induced Oral and Gastrointestinal Mucositis," Cancer, Vol. 100, Suppl. 9, 2004, pp. 2026-2046. doi:10.1002/cncr.20163

[2] E. B. Avritscher, C. D. Cooksley and L. S. Elting, "Scope and Epidemiology of Cancer Therapy-Induced Oral and Gastrointestinal Mucositis," Seminars in Oncology Nursing, Vol. 20, No. 1, 2004, pp. 3-10. doi:10.1053/j.sonen.2003.10.002

[3] L. S. Elting, C. D. Cooksley, M. S. Chambers and A. S. Garden, "Risk, Outcomes, and Costs of Radiation-Induced Oral Mucositis among Patients with Head-andNeck Malignancies," International Journal of Radiation Oncology, Biology, Physics, Vol. 68, No. 4, 2007, pp. 1110-1120. doi:10.1016/j.ijrobp.2007.01.053

[4] N. J. Nonzee, N. A. Dandade, U. Patel, T. Markossian, M. Agulnik, A. Argiris, J. D. Patel, R. C. Kern, H. G. Munshi and C. L. Bennett, "Evaluating the Supportive Care Costs of Severe Radiochemotherapy-Induced Mucositis and Pharyngitis," Cancer, Vol. 113, No. 6, 2008, pp. 14461452. doi: $10.1002 /$ encr. 23714

[5] S. T. Sonis, "Mucositis as a Biological Process: A New Hypothesis for the Development of Chemotherapy-Induced Stomatotoxicity," Oral Oncology, Vol. 34, No. 1, 1998, pp. 39-43. doi:10.1016/S1368-8375(97)00053-5

[6] C. A. Squier and M. J. Kremer, "Biology of Oral Mucosa and Esophagus," Journal of the National Cancer Institute, Vol. 29, No. 29, 2001, pp. 7-15. doi:10.1093/oxfordjournals.jncimonographs.a003443

[7] S. T. Sonis, L. S. Elting, D. Keefe, D. E. Peterson, M. Schubert, M. Hauer-Jensen, B. N. Bekele, J. Raber-Durlacher, J. P. Donnelly and E. B. Rubenstein, "Perspectives on Cancer Therapy-Induced Mucosal Injury: Pathogenesis, Measurement, Epidemiology, and Consequences for Patients," Cancer, Vol. 100, No., 2004, pp. 1995-2025. doi:10.1002/cncr.20162

[8] L. C. Pillitteri and R. E. Clark, "Comparison of a Patient-Controlled Analgesia System with Continuous Infusion for Administration of Diamorphine for Mucositis," Bone Marrow Transplantation, Vol. 22, No, 5, 1998, pp.

\section{5-498. doi:10.1038/sj.bmt. 1701370}

[9] L. S. Elting, C. Cooksley, M. Chambers, S. B. Cantor, E. Manzullo and E. B. Rubenstein, "The Burdens of Cancer Therapy: Clinical and Economic Outcomes of Chemotherapy-Induced Mucositis," Cancer, Vol. 98, No. 7, 2003, pp. 1531-1539. doi:10.1002/cncr.11671

[10] World Health Organization, "Handbook for Reporting Results of Cancer Treatment," World Health Organization, Geneva, 1979.

[11] S. M. Bentzen, M. I. Saunders, S. Dische and S. J. Bond, "Radiotherapy-Related Early Morbidity in Head and Neck Cancer: Quantitative Clinical Radiobiology as Deduced from the CHART Trial," Radiotherapy \& Oncology, Vol. 60, No. 2, 2001, pp. 123-135. doi:10.1016/S0167-8140(01)00358-9

[12] D. E. Peterson, R. J. Bensadoun and F. Roila, "On Behalf of the ESMO Guidelines Working Group, Management of Oral and Gastrointestinal Mucositis: ESMO Clinical Recommendations," Annals of Oncology, Vol. 19, Suppl. 2, 2008, pp. 122-125. doi:10.1093/annonc/mdn109

[13] S. Sonis, A. Muska, J. O'Brien, A. Van Vugt, P. LangerSafer and J. Keith, "Alteration in the Frequency, Severity and Duration of Chemotherapy-Induced Mucositis in Hamsters by Interleukin-11," European Journal of Cancer Part B: Oral Oncology, Vol. 31, No. 4, 1995, pp. 261-266. doi:10.1016/0964-1955(95)00015-A

[14] S. T. Sonis, L. Lindquist, A. Van Vugt, A. A. Stewart, K. Stam, G.-Y. Qu, K. K. Iwata and J. D. Haley, "Prevention of Chemotherapy-Induced Ulcerative Mucositis by Transforming Growth Factor Beta3," Cancer Research, Vol. 54, No. 5, 1994, pp. 1135-1138.

[15] S. T. Sonis, J. W. Costa Jr, S. M. Evitts, L. E. Lindquist and M. Nicolson, "Effect of Epidermal Growth Factor on Ulcerative Mucositis in Hamsters That Receive Cancer Chemotherapy," Oral Surgery, Oral Medicine, Oral Pathology, Vol. 74, No. 6, 1992, pp. 749-755. doi:10.1016/0030-4220(92)90402-C

[16] M. B. Tombes and B. Gallucci, "The Effects of Hydrogen Peroxide Rinses on the Normal Oral Mucosa," Nursing Research, Vol. 42, No. 6, 1993, pp. 332-337. doi:10.1097/00006199-199311000-00003

[17] R. Spielberger, P. Stiff, W. Bensinger, T. Gentile, D. Weisdorf, T. Kewalramani, T. Shea, S. Yanovich, K. Hansen and C. Emmanouilides, "Palifermin for Oral Mucositis after Intensive Therapy for Hematologic Cancers," The New English Journal of Mededicine, Vol. 351, No. 25, 2004, pp. 2590-2598. doi:10.1056/NEJMoa040125

[18] A. Dueñas-Gonzalez, P. Sobrevilla-Calvo, M. FriasMendivil, D. Gallardo-Rincon, F. Lara-Medina, L. AguilarPonce, E. Miranda-Lopez, J. Zinser-Sierra and E. Reynoso-Gomez, "Misoprostol Prophylaxis for High-Dose Chemotherapy-Induced Mucositis: A Randomized Double-Blind Study," Bone Marrow Transplantation, Vol. 17, No. 5, 1996, pp. 809-812.

[19] A. Berger, M. Henderson, W. Nadoolman, V. Duffy, D. Cooper, L. Saberski and L. Bartoshuk, "Oral Capsaicin Provides Temporary Relief for Oral Mucositis Pain Sec- 
ondary to Chemotherapy/Radiation Therapy," Journal of Pain and Symptom Management, Vol. 10, No. 3, 1995, pp. 243-248. doi:10.1016/0885-3924(94)00130-D

[20] D. E. Peterson, "Pretreatment Strategies for Infection Prevention in Chemotherapy Patients," In: NCI Monogram, Development Conference on Oral Complications of Cancer Therapies: Diagnosis, Prevention and Treatment, NCI Publication, Washington DC, 1990, pp. 61-71.

[21] H. V. Worthington, J. E. Clarkson and O. B. Eden, "Interventions for Preventing Oral Mucositis for Patients with Cancer Receiving Treatment," Cochrane Database of Systematic Reviews, Vol. 4, 2007, Article ID: CD000978.

[22] J. Bowman and M. Haas, "Supersaturated Electrolyte Oral Rinse Alleviates Painful Oral Mucositis for Patients Receiving Chemotherapy and Radiation, Oncology Nursing Forum, Vol. 35, 2008, pp. 510-511.

[23] M. Haas, T. Mercedes and M. Manyak, "Reduction in Painful Oral Mucositis by Supersaturated Calcium Phosphate Oral Rinse in Head and Neck Cancer Patients Receiving Chemotherapy and Radiation," International Journal of Radiation Oncology, Biology, Physics, Vol. 72, Suppl. 1, 2008, p. 406. doi:10.1016/j.ijrobp.2008.06.1297

[24] T. P. Bechtel and S. Devine, "Use of a Calcium/Phosphate Oral Rinse $\left(\right.$ Caphosol $\left.^{\circledR}\right)$ to Lessen the Mucositis Following Autologous Peripheral Blood Stem Cell Transplantation," Biology of Blood and Marrow Transplantation, Vol. 15, No. 2, 2009, p. 154. www.abstracts2view.com/bmt

[25] A. S. Papas, G. Koobatian, S. Samiei, E. Noto and E. Johansen, "Effects Delas Soluciones de Calico y Fosfato en Pacientes Irradiados Terapeuticamente (Parte 1)," Revista de la Asociación Odontológica Argentina, Vol. 80, 1992, pp. 88-92.

[26] A. S. Papas, E. Johansen and K. Miller, "An Oral Preventive Protocol for Bone Marrow Transplant Patients," 11th MASCC International Symposium on Supportive Care in
Cancer, Nice, 18-20 February 1999, (Abstract) CCJ11.

[27] A. S. Papas, R. E. Clark, G. Martuscelli, K. T. O’Loughlin, E. Johansen and K. B. Miller, "A Prospective, Randomized Trial for the Prevention of Mucositis in Patients Undergoing Hematopoietic Stem Cell Transplantation," Bone Marrow Transplantation, Vol. 31, 2003, pp. 705712. doi:10.1038/sj.bmt. 1703870

[28] A. Waśko-Grabowska, P. Rzepecki, S. Oborska, J. Barzał, K. Gawroński, B. Młot, C. Szczylik, "Efficiency of SuPersaturated Calcium Phosphate Mouth Rinse Treatment in Patients Receiving High-Dose Melphalan or BEAM Prior to Autologous Blood Stem Cell Transplantation: A Single-Center Experience," Transplant Proceedings, Vol. 43, No. 8, 2011, pp. 3111-3113. doi:10.1016/j.transproceed.2011.08.053

[29] A. Waśko-Grabowska, P. Rzepecki, S. Oborska, J. Barzał, B. Mlot, K. Gawroński, M. Wasko, C. Szczylik, "A Supersaturated Calcium Phosphate Solution Seems to Effectively Prevent and Treat Oral Mucositis in Haematopoietic Stem Cell Transplanted Cancer Patients-Single Centre Experience," Journal of the Balkan Union of Oncology, Vol. 17, No. 2, 2012, pp. 363-368.

[30] H. G. Wu, S. Y. Song, Y. S. Kim, Y. T. Oh, C. G. Lee, K. C. Keum, Y. C. Ahn, S. W. Lee, "Therapeutic Effect of Recombinant Human Epidermal Growth Factor (RhEGF) on Mucositis in Patients Undergoing Radiotherapy, with or without Chemotherapy, for Head and Neck Cancer: A Double-Blind Placebo-Controlled Prospective Phase 2 Multi-Institutional Clinical Trial," Cancer, Vol. 115, No. 16, 2009, pp. 3699-3708. doi:10.1002/cncr.24414

[31] Q. T. Le, H. E. Kim, C. J. Schneider, G. Muraközy, K. Skladowski, S. Reinisch, Y. Chen, M. Hickey, M. Mo, M. G. Chen, D. Berger, R. Lizambri and M. Henke, "Palifermin Reduces Severe Mucositis in Definitive Chemoradiotherapy of Locally Advanced Head and Neck Cancer: A Randomized, Placebo-Controlled Study," Journal of Clinical Oncology, Vol. 29, No. 20, 2011, pp. 2808-2814. doi:10.1200/JCO.2010.32.4095 